\title{
Previsão de Longo Prazo da Umidade do Solo no Rio Uruguai
}

\author{
Claudinéia Brazil Saldanha, Carlos E. M. Tucci \\ Instituto de Pesquisas Hidráulicas - IPH/UFRGS \\ neiabrazil@yahoo.com.br, cemtucci@uol.com.br \\ Eric F. Wood, Haibin Li \\ Environmental Engineering and Water Resources, Department of Civil and Environmental Engineering \\ Princeton University, USA
}

Recebido: 20/03/11 - revisado: 25/06/11 - aceito: 02/08/12

\begin{abstract}
RESUMO
A previsão da umidade do solo é necessária para o planejamento agrícola e para o mercado de commodities agrícolas. Esta previsão é realizada em longo prazo (sazonal) com antecedência de alguns meses. Este tipo de estimativa apresenta um grau de incerteza que depende da previsão dos modelos climáticos. Neste estudo a previsão em longo prazo da umidade do solo foi desenvolvida a partir das previsões de precipitação do modelo CFS (Climate Forecast System) com o horizonte de até 6 meses para parte da bacia do rio Uruguai no Rio Grande do Sul. A umidade do solo foi estimada com base no módulo de balanço de água no solo do modelo hidrológico MGB-IPH. Os resultados obtidos mostraram que a metodologia proposta apresenta melhores resultados de previsão com relação aos métodos atualmente existentes, considerando a redução do erro padrão da estimativa com antecedências de 1 a 6 meses. Estes resultados permitem melhorar a estimativa da umidade do solo e da produtividade agrícola em sub-regiões da bacia do rio Uruguai no Rio Grande do Sul com as antecedências mencionadas.
\end{abstract}

Palavras-chave: Umidade do sol. Previsão climática. Modelo hidrológico.

\section{INTRODUÇÃO}

As condições climáticas e do solo são fatores importantes para o planejamento agrícola. A umidade do solo é amplamente utilizada como uma variável em muitos estudos ambientais, na hidrologia, meteorologia e agricultura (Walker, 2004). O conhecimento da distribuição da umidade do solo e sua ligação com a evaporação e transpiração são essenciais para prever a influência das condições da superfície terrestre nos processos de tempo e clima. De acordo com Lopes et al. (2011) a umidade do solo influencia a separação da precipitação em armazenamento e escoamento, possuindo uma ligação direta com os fluxos de calor sensivel e latente, que consequentemente influenciam a taxa de evapotranspiração, contribuindo para o crescimento da cultura.

O acompanhamento da umidade do solo no espaço e no tempo permite uma melhor gestão dos recursos hídricos, previsão de inundações, previsão de seca entre outros. Ao contrário da vazão e das variáveis climáticas, apesar de sua importância a umidade do solo não é monitorada regularmente. A falta de acompanhamento desta variável é devido ao alto custo do monitoramento, e mesmo que houvesse este monitoriamento, seria muito difícil obter uma boa resolução espacial e temporal (CHEN e HU, 2004).

A umidade do solo é uma das variáveis climáticas que mais influenciam a produção agrícola. O conhecimento antecipado desta variável contribuiria para melhor definição de quando plantar e estimar a produtividade das culturas, permitindo reduzir as incertezas na tomada de decisão do planejamento das atividades agrícolas e fornecendo subsídio sobre: (a) o potencial impacto sobre as culturas escolhidas; (b) demanda de rega, se houver disponível; (c) produtividade; e (d) investimento efetivo no plantio.

Existem alguns métodos tradicionais para cálculo da umidade do solo (SILVA, 2001) dos quais se destaca o proposto por Thornthwaite \& Mather (1955), porém é muito simplificado, os parâmetros são estimados com base nos valores médios mensais de temperatura e total de precipitação (ROSSATO, et al., 2004; CARDOSO et al., 2003). 
Para determinar a umidade do solo com antecedência de 1 a 6 meses, é necessário prever o valor das variáveis climáticas utilizadas no seu cálculo. Estudos recentes mostraram resultados promissores neste tipo de previsão (TUCCI et al., 2002; BEZERRA, 2006).

Este artigo apresenta resultados da previsão em longo prazo da umidade do solo (sazonal) baseado num modelo climático e num modelo hidrológico de balanço de água no solo.

\section{METODOLOGIA}

A metodologia consiste no uso de um modelo climático para estimativa da precipitação e um modelo hidrológico de balanço de água que estima a umidade do solo com base nas variáveis climáticas e no tipo de solo da região de estudo. Considerando que não existem dados de umidade do solo, os resultados foram avaliados com base na estimativa da umidade determinada a partir da precipitação observada, chamada umidade de aferição.

\section{Modelo hidrológico de balanço da água no solo}

O modelo de previsão de umidade do solo (WBMGB) proposto por Saldanha (2009), é um modelo distribuído, constituído por uma malha regular, dividida em células quadradas de aproximadamente $10 \times 10 \mathrm{~km}$, sendo composto por 4 rotinas principais: Escoamento superficial, escoamento sub-superficial, escoamento subterrâneo e fluxo ascendente de água no solo. Este modelo é baseado no módulo de balanço de água do solo do Modelo de Grandes Bacias do IPH (MGB-IPH) (COLLISCHONN, 2001), calculado a partir da seguinte equação com intervalo de tempo mensal:

$W_{t}=W_{t-1}+P-E T-D_{S U P}-D_{I N T}-D_{B A S}+D_{c a p}$

em que Wt - armazenamento na camada de solo ao longo do intervalo de tempo $\mathrm{t}[\mathrm{mm}] ; \mathrm{W}_{\mathrm{t}-1}$ - o armazenamento na camada de solo no intervalo de tempo anterior (t-1) [mm]; P - precipitação ao longo do intervalo de tempo [mm]; ET - evapotranspiração da água do solo ao longo do intervalo de tempo [mm]; e $D_{\text {SUP }}$ - escoamento superficial ao longo do intervalo de tempo (drenagem rápida) [mm]. Além do escoamento superficial, o solo é drenado pelo escoamento subterrâneo $\left(\mathrm{D}_{\mathrm{BAS}}\right)$, e pelo escoamento subsuperficial $\left(\mathrm{D}_{\mathrm{INT}}\right)$ que é o escoamento menos rápido do que o superficial, porém mais rápido do que o subterrâneo. Em algumas situações a camada de solo também pode receber um fluxo de umidade ascendente denominado $\mathrm{D}_{\mathrm{CAP}}$.

A precipitação utilizada para o cálculo da umidade do solo é obtida descontando a precipitação incidente da taxa de interceptação, essa taxa depende do índice de área foliar (IAF), obtido a partir de valores médios mensais.

De acordo com Collischonn (2001) uma fração da precipitação, que depende do estado de armazenamento de água na camada de solo (W), e do armazenamento máximo na camada de solo $\left(\mathrm{W}_{\mathrm{m}}\right)$, é imediatamente transformada em escoamento superficial $\left(\mathrm{D}_{\text {SUP }}\right)$. Para calcular o escoamento superficial é necessário fazer o seguinte teste:

$\delta=\left[\left(1-\frac{W}{W_{m}}\right)^{\frac{1}{b+1}}-\frac{P}{(b+1) \cdot W_{m}}\right]$

Quando $\delta \leq 0$ o escoamento superficial é calculado por:

$D_{\text {sup }}=P-\left(W_{m}-W\right)$

quando $\delta>0 \quad$ o escoamento superficial é calculado por:

$D_{\text {sup }}=P-\left(W_{m}-W\right)+W_{m} \cdot(\delta)^{b+1}$

onde $\mathrm{W}$ - armazenamento na camada do solo [mm]; $\mathrm{W}_{\mathrm{m}}$ - armazenamento máximo na camada do solo [mm]; b - parâmetro adimensional que representa a não-uniformidade da capacidade de armamento do solo no bloco é um parâmetro calibrável que depende do tipo de uso do solo.

$\mathrm{O}$ escoamento sub-superficial $\left(\mathrm{D}_{\mathrm{INT}}\right)$ é obtido por uma relação não linear com o armazenamento na camada de solo (Equação 5).

$D_{I N T}=\left[K_{I N T}\left(\frac{W-W_{Z}}{W_{m}-W_{Z}}\right)^{\frac{3+2}{X L}}\right]$

em que $\mathrm{W}_{\mathrm{z}}$ - limite de armazenamento para haver escoamento sub-superficial $[\mathrm{mm}] ; \mathrm{K}_{\mathrm{INT}}$ - parâmetro de escoamento sub-superficial [mm]; XL - índice de porosidade do solo (parâmetro adimensional). O parâmetro $\mathrm{K}_{\mathrm{INT}}$ é calibrado e o índice XL é fixado 
em 0,4, que é uma média para diferentes tipos de solo da região de estudo.

$\mathrm{O}$ escoamento subterrâneo $\quad\left(\mathrm{D}_{\mathrm{BAS}}\right)$ é calculado por uma equação simples, linear com relação ao armazenamento no solo.

$D_{B A S}=K_{B A S}\left(\frac{W-W_{C}}{W_{m}-W_{c}}\right)$

onde $\mathrm{W}_{\mathrm{c}}$ - limite de armazenamento no solo para haver escoamento subterrâneo $[\mathrm{mm}]$; $\mathrm{K}_{\mathrm{BAS}}$ parâmetro de escoamento subterrâneo [mm].

Quando W é menor do que $W_{z}$, não há escoamento sub-superficial, e quando W é menor do que $\mathrm{W}_{\mathrm{c}}$, não há escoamento subterrâneo.

Em alguns casos, quando o armazenamento do solo é baixo, pode ocorrer a transferência de água do reservatório subterrâneo para a camada de solo. Esta possibilidade visa permitir ao modelo simular situações em que as águas subterrâneas voltem a ser disponibilizadas para a evapotranspiração. A Equação 7 descreve o fluxo ascendente.

$D_{\text {cap }}=\frac{W_{C}-W}{W_{c}} D M_{c a p}$

onde: $\mathrm{D}_{\text {cap }}$ - fluxo ascendente $[\mathrm{mm}] ; \mathrm{DM}_{\text {cap }}$ - máximo fluxo ascendente para o solo (parâmetro do modelo) $[\mathrm{mm}]$.

Para o cálculo das equações descritas anteriormente é necessário estimar os seguintes parâmetros: $\mathrm{W}_{\mathrm{m}}, \mathrm{W}_{\mathrm{z}}, \mathrm{W}_{\mathrm{c}}, \mathrm{b}, \mathrm{XL}, \mathrm{K}_{\mathrm{int}}$ e $\mathrm{K}_{\mathrm{bas}}$

Os parâmetros podem ser medidos, ajustados ou são poucos sensíveis (Collischonn e Tucci, 2005; Tucci, et. al., 2002). De acordo com Collischonn (2001) os parâmetros de armazenamento residual Wz e Wc podem ser assumidos como $10 \%$ do Wm., para evitar a possibilidade de escoamento negativo.

Para o índice de distribuição do tamanho dos poros $(\mathrm{Xl})$, adotou-se o valor de 0,4 que corresponde à média dos solos da região de estudo. Os parâmetros Kint, o Kbas e o b foram determinados anteriormente (SALDANHA, 2009) e podem ser observados na Tabela 1.

A determinação da capacidade máxima de armazenamento de água no solo (Wm) foi desenvolvida a partir dos mapas de solo elaborados pelo IBGE (Instituto Brasileiro de Geografia e Estatística) e pela Embrapa (Empresa Brasileira de Pesquisa Agropecuária) com uma escala de 1:5.000.000 a partir de levantamentos de solos produzidos pelo Projeto
RADAMBRASIL. Os principais tipos de solos encontrados na região, originados de basalto, ocorrendo, especialmente solos do tipo: Cambissolo Háplico, Latossolo Bruno, Latossolo Vermelho, Neossolo Litólico e o Nitossolo Vermelho.

Tabela 1 - Valores dos parâmetros associados a cada subbacia do rio Uruguai.

\begin{tabular}{c|c|ccc}
\hline & & \multicolumn{3}{|c}{ Parâmetros } \\
Sub-bacias & $\begin{array}{c}\text { Área } \\
\left(\mathrm{km}^{2}\right)\end{array}$ & $\mathrm{b}$ & $\begin{array}{c}\text { Kint } \\
(-)\end{array}$ & $\begin{array}{c}\text { Kbas } \\
(\mathrm{mm})\end{array}$ \\
\hline Rio Ijui & 9.452 & 0,72 & 117,2 & 5,95 \\
Rio Comandaí & 1.417 & 0,30 & 45,2 & 1,15 \\
Rio Santa Rosa & 1.310 & 0,27 & 207,2 & 2,34 \\
Rio Buricá & 2.185 & 0,45 & 47,2 & 3,0 \\
Rio Turvo & 1.531 & 0,23 & 220 & 4,41 \\
Rio Guarita & 2.078 & 0,31 & 240,2 & 6,34 \\
Rio da Várzea & 5.352 & 0,10 & 10,2 & 1,54 \\
Rio Passo Fundo & 3.714 & 0,04 & 1,5 & 0,13 \\
Rio Apuaê & 3.710 & 0,29 & 73,4 & 2,66 \\
Rio Forquilha & 1.634 & 0,13 & 138,2 & 1,55 \\
\hline
\end{tabular}

A Tabela 2 mostra os valores $\mathrm{Wm}$ adotados nesta pesquisa para cada tipo de solo, estes valores foram estimados conforme a textura do solo, utilizando como subsídios os valores tabelados de Thornthwaite \& Mather (1957).

Tabela 2 - Capacidade máxima de armazenamento (Wm)dos solos da área de estudo.

\begin{tabular}{ll}
\hline Classe de solo & Wm $(\mathrm{mm})$ \\
\hline Cambissolo Háplico & 200 \\
Latossolo Bruno & 260 \\
Latossolo Vermelho & 350 \\
Neossolo Litólico & 100 \\
Nitossolo Vermelho & 350 \\
\hline
\end{tabular}

\section{Previsão Climática}

Nesta pesquisa, foram utilizadas as previsões de longo prazo do modelo global CFS (Climate forecast system) do NCEP (National Centers for Environmental Prediction), realizadas com periodicidade mensal para o horizonte de 6 meses. Para aumentar a eficiência do modelo foi utilizada a previsão por conjunto que consiste em construir um conjunto de possíveis estados iniciais, ligeiramente diferentes entre si, integrando o modelo numérico a partir de cada estado inicial, produzindo um con- 
junto de previsões. As séries geradas são denominadas membros do modelo.

A resolução do modelo é de aproximadamente $250 \mathrm{~km}$ (SAHA et al., 2006; YUAN et al., 2011), foram utilizados 648 pontos de grade para a região de estudo e a média dos 14 membros. Estas previsões foram fornecidas pelo professor Eric Wood do Departamento de Engenharia Civil e Ambiental da Universidade de Princeton, New Jersey, EUA.

As séries de previsões de precipitação foram fornecidas com as respectivas correções pela técnica Bayesiana para minimizar os erros sistemáticos do modelo. Dentro da estrutura bayesiana, a distribuição climatológica da variável é fundamental sendo utilizada juntamente com o modelo climático para obter a equação de regressão linear ponderada entre o modelo e a variável climática. De acordo com Luo et al. (2007), após a correção da série de dados prevista, o modelo é capaz de representar melhor a estimativa da variável, incluindo sua média e variância.

As previsões de precipitação do modelo global do NCEP, foram interpoladas e utilizadas como dados de entrada para o modelo de balanço de água no solo, gerando previsões de umidade do solo com até 6 meses de antecedência, em intervalo de tempo mensal. Este tipo de previsão se baseia no conhecimento recente do clima para prognosticar as variáveis climáticas dos meses subsequentes.

\section{Localização}

O estudo foi realizado em uma área compreendida entre as coordenadas de $-27^{\circ} 50^{\prime}$ e $-29^{\circ} 00^{\prime}$ de latitude e $-55^{\circ} 00^{\prime}$ e $-51^{\circ} 50^{\prime}$ de longitude. A área faz parte da Bacia do rio Uruguai e localiza-se no Estado do Rio Grande do Sul e é caracterizada pela intensa produção agrícola. O rio Uruguai é, junto com o rio Paraná, um dos maiores formadores do rio da Prata.

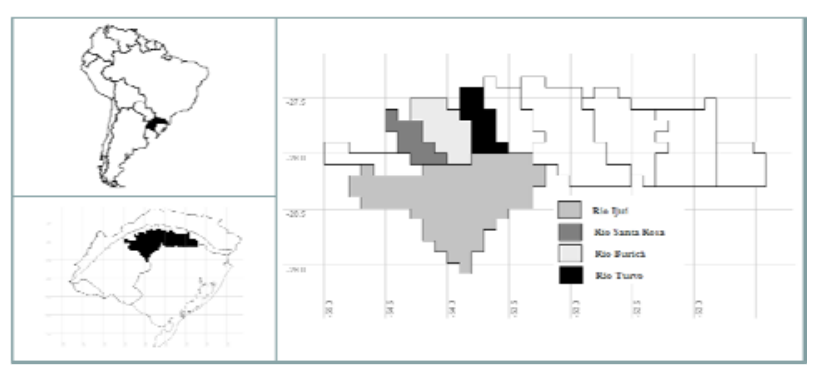

Figura 1 - Localização da área de estudo.
$\mathrm{O}$ rio Uruguai forma-se na região sul do Brasil, entre os Estados de Santa Catarina e do Rio Grande do Sul, a partir da confluência dos rios Canoas e Pelotas. A área da bacia em território brasileiro é de $177.494 \mathrm{~km} 2$ (TUCCI, et al., 2002). A área considerada para a análise realizada neste trabalho envolve apenas as sub-bacias do rio Ijuí, rio Santa Rosa, rio Buricá e rio Turvo.

\section{Agricultura da região}

A cultura selecionada para o estudo foi a soja, que é uma cultura de verão, sendo a umidade do solo um fator importante para seu desenvolvimento, pois quando o solo está muito seco, ele dificulta a maturação e a colheita e a umidade excessiva pode trazer prejuízos à lavoura (CAMARGO et. al., 1974).

A produtividade agrícola depende do clima e de sua variabilidade, e esta dependência é importante durante o ciclo de vida de determinadas culturas, sendo a responsável pela alternância de suas produções anuais, influenciando diretamente no balanço de produção, havendo perdas ou ganhos em função das condições climáticas de uma região (FERREIRA, 2006). As relações entre os elementos climáticos e a produção agrícola são bastante complexas, pois os fatores do ambiente podem afetar o crescimento e o desenvolvimento das plantas sob diferentes formas nas diversas fases do ciclo da cultura.

De acordo com Berlato e Fontana (1999), os maiores rendimentos da soja da década de 1990 foram em anos de El Niño (1991/1992, 1992/1993, 1993/1994, 1994/1995 e 1997/1998), que determinaram precipitações pluviais acima da média climatológica. No evento de El Nino de 2002/2003 a soja apresentou rendimento recorde no Estado do Rio Grande do Sul de $2.600 \mathrm{~kg} / \mathrm{ha}$.

O ciclo de vida de uma planta pode ser dividido em dois estádios principais: vegetativo e reprodutivo. O estádio vegetativo compreende a emergência da plântula e o crescimento da planta com o consequente aumento da fitomassa. O estádio reprodutivo compreende a emissão de flores, a formação de vagens, o enchimento dos grãos e a maturação. O tempo de duração em todos sub-períodos de desenvolvimento não é fixo e pode variar mais ou menos entre genótipos com ciclos diferentes (ALMEIDA, 2005).

A época de semeadura da soja pode ser alterada quando há um bom regime de precipitação e fertilidade elevada do solo. De um modo geral, o período de semeadura em que a produtividade da soja tende a ser elevada abrange os meses de outu- 
bro a dezembro, porém novembro corresponde ao mês preferencial de plantio (FERREIRA, 2006; FERREIRA et al., 2010).

De acordo com Fendrich (2003) a planta é sensível às condições de solo saturado, por isso ele deve ser bem drenado e arejado. O sistema radicular da soja é bem superficial. Medidas feitas indicam que cerca de $80 \%$ do seu peso se encontra na camada $0-15 \mathrm{~cm}$ mais de $90 \%$, na zona arável $(0-30 \mathrm{~cm})$. A raiz, em condições favoráveis, pode alcançar profundidades maiores que um metro. As mais profundas, apesar de em menores quantidades, parecem ter uma atividade importante. Perfis de umidade de solo indicam que até $50 \mathrm{~cm}$ de profundidade, a extração de água pela soja é muito grande, vindo desta camada cerca de 80 a $90 \%$ da evapotranspiração real. Dentro de certos limites, a soja mostra uma notável capacidade de se adaptar a um déficit de água.

\section{Umidade do solo de aferição}

Na região de estudo não existem dados medidos de umidade do solo, portanto para verificação dos resultados, foi gerado um banco de dados de umidade de aferição calculado através do modelo de balanço de água no solo a partir da precipitação observada.

A capacidade do modelo em prever a umidade do solo foi verificada com dados observados por Saldanha (2009) em outra região.

Após a obtenção dos valores previstos e da série de umidade de aferição, foram construídos os intervalos de confiança para cada horizonte de previsão, para todo o período de estudo de janeiro de 1995 a dezembro de 2005, gerados por 1000 iterações, com $95 \%$ de confiança, utilizando o método Bootstrap. Com a banda de confiança é possível verificar o desempenho global do modelo estudado.

A técnica de reamostragem Bootstrap desenvolvida por Efron e Tibshirani (1993) permite precisamente usar uma amostra para estimar a quantidade de interesse através de uma estatística e avaliar também as propriedades da distribuição dessa estatística.

O método bootstrap obtém sua amostra via amostragem com reposição da amostra original. A chave é a reposição das observações após a amostragem, o que permite ao pesquisador criar tantas amostras quanto necessárias e jamais se preocupar quanto à duplicação de amostras, exceto quando isso acontecer ao acaso. Cada amostra pode ser analisada independentemente e os resultados compila- dos ao longo da amostra. Por exemplo, a melhor estimativa da média populacional é exatamente a média de todas as médias estimadas ao longo das amostras (COSTA, 2010).

\section{RESULTADOS}

\section{Previsões}

As simulações foram feitas para o período de janeiro de 1994 a dezembro de 2005, onde o primeiro ano foi descartado da análise dos resultados por ter sido utilizado para o aquecimento do modelo.

As previsões de umidade do solo foram desenvolvidas para os horizontes de 1, 3 e 6 meses. Foram disponibilizados para esta pesquisa 14 membros de previsão de precipitação do modelo global CFS, sendo utilizada a média de todos os membros (previsão por ensemble).

A Figura 2 mostra a previsão de umidade do solo com seus respectivos intervalos de confiança, a linha com marcadores representa a umidade do solo prevista; a linha continua representa a umidade do solo de aferição; e a área cinza, a banda de confiança calculada a partir dos membros gerados pelo modelo de previsão para o horizonte de 6 meses de previsão.

Com base na Figura 2 é possível fazer uma análise visual da umidade do solo prevista e da umidade do solo de aferição verificando que as previsões de umidade do solo com 6 meses de antecedência representaram satisfatoriamente a curva da umidade do solo de aferição, com valores dentro da banda de confiança.

A Figura 3 apresenta os resíduos das previsões para as bacias estudadas, para verificar se o modelo proposto apresentou ganho em relação as técnicas atualmente existentes, a umidade prevista foi comparada com a umidade do solo calculada a partir das normais climatológicas, ou seja com as médias de longo período da precipitação, assumindo os valores médios para todos os meses do ano do período estudado.

Avaliando os resíduos do modelo na bacia do rio Ijuí, verificou-se que $75 \%$ dos valores observados para todos os horizontes de previsão, ficaram próximos a zero, enquanto que no método tradicional ficaram em torno de $-100 \mathrm{~mm}$, indicando uma forte subestimativa entre os valores previstos e observados. 


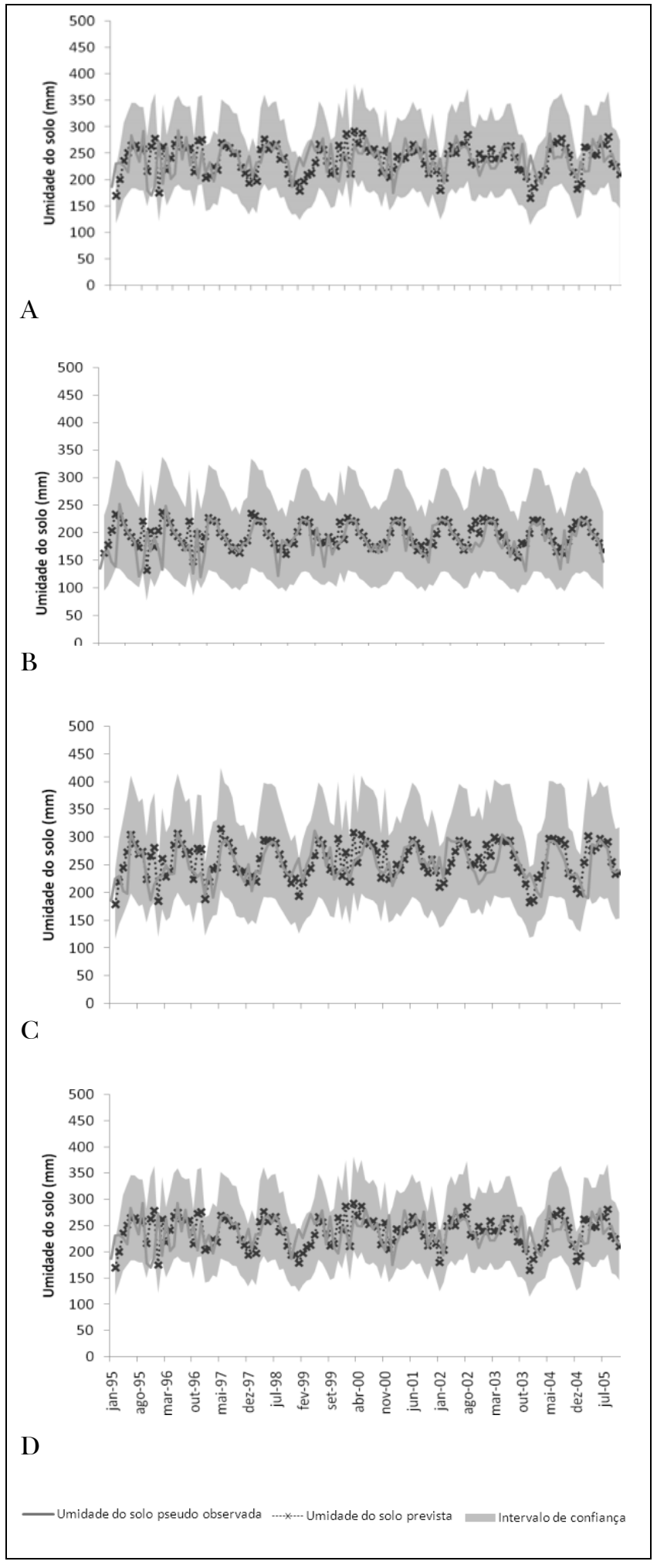

Figura 2 - Previsão de umidade do solo com intervalo de confiança para o horizonte de 6 meses. Bacia do rio Ijuí (a), Bacia do rio Santa Rosa (b), bacia do rio Buricá (c) e bacia do rio Turvo.

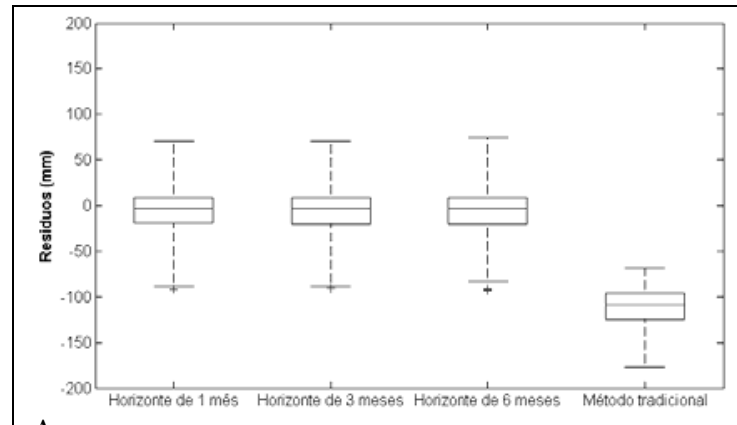

A

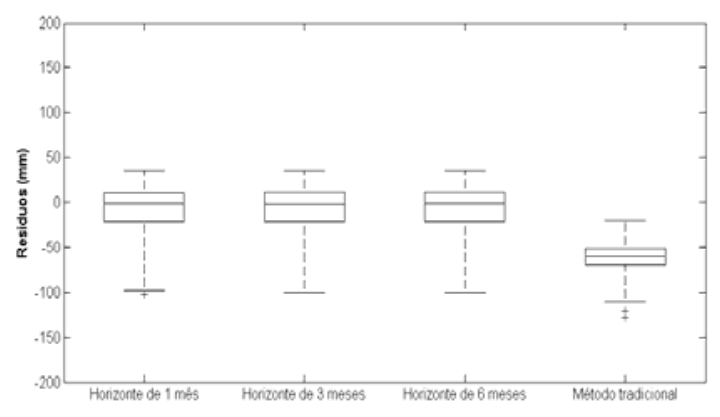

B

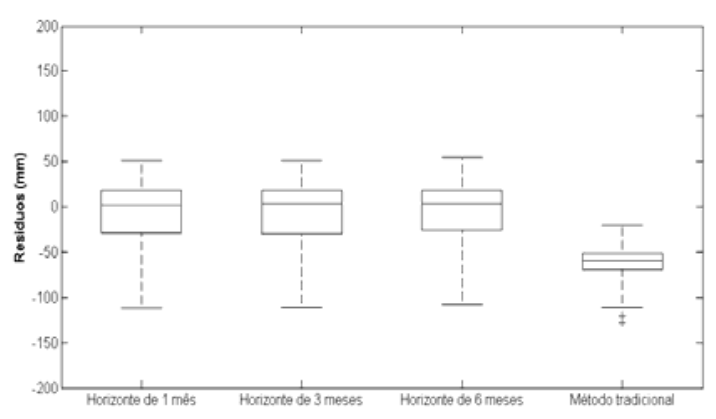

$\mathrm{C}$

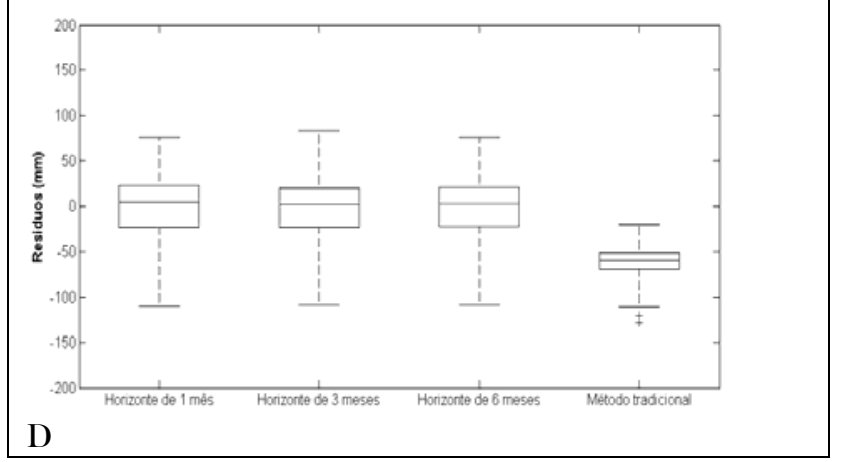

Figura 3 - Resíduos do modelo de previsão de umidade do solo (diagrama de caixa)- A linha horizontal no interior das caixas representa o percentil 50 (mediana); o final das caixas, os percentis 25 e 75; as barras, os percentis $10 \mathrm{e}$ 90; o símbolo de cruz representa os valores extremos.

Bacia do rio Ijuí (a), Bacia do rio Santa Rosa (b), bacia do rio Buricá (c) e bacia do rio Turvo. 
A bacia do rio Santa Rosa apresentou um comportamento similar a do rio Ijuí, enquanto nas demais bacias $75 \%$ dos resíduos ficaram em torno de $10 \mathrm{~mm}$, indicando ainda um bom desempenho do modelo proposto, com relação ao método tradicional todas as bacias apresentaram uma forte subestimativa entre a umidade do solo prevista e a calculada.

No geral os gráficos apresentados mostram que a maior parte dos erros absolutos da umidade do solo prevista situa-se na faixa entre -30 e $10 \mathrm{~mm}$, o que significa que a maioria dos erros é pequena. A umidade do solo calculada a partir do método tradicional apresentou erros superiores a $-50 \mathrm{~mm}$, mostrando uma tendência do método tradicional em subestimar a umidade do solo observada. Verificouse também que os resíduos do modelo proposto não apresentaram diferenças significativas entre os horizontes de previsão avaliados, ou seja, para 1, 3 e 6 meses de antecedência.

Portanto, a metodologia proposta pode servir como subsidio para o planejamento agrícola, principalmente no caso de culturas que dependam do conteúdo disponível de água no solo para o seu desenvolvimento, sendo possível fornecer previsões de umidade do solo mais confiáveis, com até 6 meses de antecedência.

\section{CONCLUSÕES}

Esta análise baseou-se principalmente na capacidade do modelo climático em prever com antecedência a precipitação que permite a estimativa da umidade do solo. Utilizaram-se dois cenários: a umidade do solo estimada pela previsão da chuva com antecedência e a umidade do solo estimada com a precipitação observada para aferição. $\mathrm{O}$ desempenho do modelo de previsão em simular a umidade do solo foi satisfatório em comparação com os métodos atualmente existentes.

Avaliando os resíduos do modelo de previsão, concluiu-se que a previsão de umidade do solo a partir do método tradicional, ou seja, calculado a partir da média de longo período da precipitação, subestima claramente a umidade do solo de aferição, enquanto o erro das previsões para os horizontes analisados foi pequeno. Portanto, o método proposto apresenta ganhos em relação ao método tradicional.

A conclusão final que se obtém por este estudo é que o acoplamento dos dados dos modelos atmosféricos no modelo de previsão de umidade do solo se demonstrou satisfatório comparado com a metodologia atualmente existente, podendo trazer benefícios para o plantio de sequeiro, permitindo o planejamento mais adequado e a redução de prejuízos.

\section{AGRADECIMENTOS}

Os autores agradecem ao $\mathrm{CNPq}$ pela concessão de bolsa de doutorado ao primeiro autor e a Universidade de Princeton pela disponibilização dos dados.

\section{REFERÊNCIAS}

ALMEIDA, I. R. O clima como fator de expansão da cultura da soja no Centro-Oeste. 2005. Tese (Doutorado) - Universidade Estadual Paulista, Faculdade de Ciências e Tecnologia, 2005.

BERLATO, M. A., FONTANA, D.C. Variabilidade interanual de chuva pluvial e rendimento de soja no Estado do Rio Grande do Sul. Revista Brasileira de Agrometeorologia, Santa Maria, v.7, n.1, p.119-125, 1999.

BEZERRA, A. C. N. Aspectos da circulação atmosférica de grande escala sobre o norte e nordeste do Brasil relacionados com a temperatura da superfície do mar. 2006. Dissertação (Mestrado) - Universidade Federal de Campina Grande, 2006.

CAMARGO, A.P.; PINTO, H.S.; PEDRO JR., M.J. Aptidão climática de culturas agrícolas. In: São Paulo, Secretaria da Agricultura. Zoneamento Agrícola do Estado de São Paulo. São Paulo, v.1, p.109-149, 1974.

CARDOSO, G. O., ULLMANN, M. N., EBERHARDT, E. L. Balanço Hídrico Agro-Climático para Lages-SC. Revista de Ciencias Agroveterinarias. v. 2, n.2, p. 118-130, 2003.

COLLISCHONN, W. Simulação hidrológica de grandes bacias. 2001. Tese (Doutorado). IPH - Universidade Federal do Rio Grande do Sul, 2001.

COLlischOnN, W. TUCCI, C. E. M. Previsão Sazonal de Vazão na Bacia do Rio Uruguai 1: Ajuste e 
Verificação do Modelo Hidrológico Distribuído. Revista Brasileira de Recursos Hidricos v. 10, n. 4, p. 4359, 2005.

COSTA, G. G. O. Intervalo de confiança e teste de significância bootstrap para coeficiente de correlação linear referente à hipótese de um valor não nulo. Gestão da Produção, Geração e Sistemas. Ano 5, n. 2, p. 177-186, 2010.

CHEN, X., HU, Q. Groundwater influences on soil moisture and surface evaporation. Journal of Hydrology, v. 297, p. $285-300,2004$.

EFRON, B., TIBSHIRANI, R. J. An Introduction to the Bootstrap. New York: Chapman and Hall, 1993.

FENDRICH, R. Chuva e produtividade da soja na Fazenda Experimental Gralha Azul da PUCPR Revista Acadêmica: ciências agrárias e ambientais, Curitiba, v.1, n.2, p. 37-46, 2003.

FERREIRA, D. B. Relação entre a variabilidade da precipitação e a produtividade agrícola de soja e milho nas regiões sul e sudeste do Brasil. 2006. Dissertação (Mestrado). INPE-São Paulo, 2006.

FERREIRA JUNIOR, J. A., ESPINDOLA, S. M. C. G., GONÇALVES, D. A.R., LOPES, E. W. Avaliação de Genótipos de Soja em diferentes épocas de plantio e densidade de semeadura no município De Uberaba - MG. FAZU. Revista Uberaba, n.7, p. 13- 21, 2010.

LOPES, H. L., ACCIOLY, L. O., SILVA, F., SOBRAL, M. C., ARAUJO FILHO, J. CANDEIAS, A. L. B. Espacialização da umidade do solo por meio da temperatura da superfície e índice de vegetação. Revista Brasileira de Engenharia Agrícola Ambiental, v.15, n. 9, p. 973-980, 2011.

LUO L., E. F. WOOD, AND M. PAN. Bayesian Merging Of Multiple Climate Model Forecasts for Seasonal Hydrological Predictions. Journal Of Geophysical Research, v. 112, D10102, 2007. Doi:10.1029/2006JD007655.'

ROSSATO, L., ALVALÁ, R. C. S., TOMASELLA, J. Variação espaço-temporal da umidade do solo no Brasil: Análise das condições médias para o período de 1971-1990. Revista Brasileira de Meteorologia, v.19, n.2, 113-122, 2004.

SAHA, S., NADIGA, S., THIAW, C., WANG, J., WANG, J., ZHANG, Q., VAN DEN DOOL, H. M.,
PAN, H., MOORTHI, S., BEHRINGER, STOKES, D., WHITE, G., LORD, S., EBISUZAKI, W., PENG, P., XIE, P. The NCEP Climate Forecast System. Journal of Climate, v. 19, n. 15, p. 3483-3517, 2006.

SALDANHA, C. B. Previsão de longo prazo da umidade do solo na bacia do rio Uruguai. 2009. Tese (Doutorado). IPH - Universidade Federal do Rio Grande do Sul, 2009.

SILVA, M. A. V. Meteorologia e Climatologia. INMET/Brasilia-DF, 2001.

THORNTHWAITE, C.W.. MATHER, J. R. The water balance. Publications in Climatology. New Jersey: Drexel Institute of Technology, 1955. 104p.

THORNTHWAITE, C. W.; MATHER, J. R. Instructions and tables for computing potential evapotranspiration and the water balance. Publications in clima tology. Centerton, New Jersey, v. 10, n. 3, p. 181-311, 1957.

TUCCI, C. E. M.; CLARKE, R. T.; DIAS, P. L. S. COLLISCHONN, W. Previsão de Médio Prazo da Afluência de Reservatórios com Base na Previsão Climática. IPH-UFRGS/ IAG-USP/ANEEL/CPTECINPE. Projeto: BRA/00/029. Relatório Final, 2002.

WALKER, J.P., WILLGOOSE, G.R., KALMA, J.D. In situ measurement of soil moisture: a comparison of techniques. Journal of Hydrology, v. 293, p.85 - 99, 2004.

YUAN, X., WOOD, E. F., LUO, L., PAN, M. A first look at Climate Forecast System version 2 (CFSv2) for hydrological seasonal prediction. Geophysical Research Letters, v. 38. L13402, 2011.

\section{Long Term Forecast of Soil Moisture in the Uruguay River}

\section{ABSTRACT}

The long term forecast of Soil moisture was developed based on CFS precipitation models (Climate Forecast System) with a horizon of up to 6 months and estimated soil moisture based on the module of water balance in the soil of the hydrological model MGB-IPH. The results showed that the proposed methodology in this research provides the best prediction results compared to the currently applied methods, considering the reduction of the standard error of estimate in a horizon from 1 to 6 months. These 


\section{RBRH - Revista Brasileira de Recursos Hídricos Volume 17 n.4 - Out/Dez 2012, 39-47}

results allow better estimation of soil moisture and agricultural productivity in sub-regions of the Uruguay river basin in Brazil.

Key-words: soil moisture, climate forecast, hydrologic model. 